\title{
Eigenvalue correlations and the distribution of ground state angular momenta for random many-body quantum systems
}

\author{
J. Barea* \\ Center for Theoretical Physics, Sloane Physics Laboratory, Yale University, P. O. Box 208210, New Haven, Connecticut 06520-8120, USA \\ R. Bijker and A. Frank \\ Instituto de Ciencias Nucleares, Universidad Nacional Autónoma de México, A. P. 70-543, 04510 México, D. F., México
}

(Received 7 November 2008; published 4 May 2009)

\begin{abstract}
The observed preponderance of ground states with angular momentum $L=0$ in many-body quantum systems with random two-body interactions is analyzed in terms of correlation coefficients (covariances) among different eigenstates. It is shown that the geometric analysis of Chau et al. can be interpreted in terms of correlations (covariances) between energy eigenvalues, thus providing an entirely statistical explanation of the distribution of ground state angular momenta of randomly interacting quantum systems that, in principle, is valid for both fermionic and bosonic systems. The method is illustrated for the interacting boson model.
\end{abstract}

DOI: 10.1103/PhysRevC.79.054302 PACS number(s): 21.10.-k, 05.30.-d, 21.60.-n, 24.60.Lz

\section{INTRODUCTION}

Low-lying spectra of many-body quantum systems often display a high degree of order and regularity. In the case of atomic nuclei, despite their complexity and the large number of degrees of freedom involved, they often exhibit simple features, such as pairing properties, surface vibrations, and rotational motion in even-even nuclei. Conventional wisdom is that regularities arise from symmetries of the Hamiltonian, which lead to invariances that severely constrain the many-body motion. While some of these symmetries are exact (e.g., rotational and reflection invariance), others are approximate (e.g., isospin). These global symmetries, however, do not explain by themselves the regular patterns observed. Further assumptions about the nature of the nucleonnucleon interaction are required. Thus, a strongly attractive pairing force between like nucleons has been shown to be responsible for the remarkable constancy of the excitation energy of the first excited $L=2$ states in the $\mathrm{Sn}$ isotopes, while deformation and rotational behavior is known to arise from an attractive quadrupole-quadrupole interaction between neutrons and protons (see, e.g., Ref. [1]). These striking patterns as well as many other correlations have been shown to be robust features of low-energy nuclear behavior, which signal the emergence of order and collectivity. In every case, the patterns arise as a consequence of particular forms of the nucleon-nucleon interaction. Most features of low-lying nuclear spectra have thus been explained in terms of a short-range pairing interaction and a long-range quadrupole force.

Hence, it came as a big surprise when Johnson, Bertsch, and Dean [2] found that for even-even nuclei, shell-model Hamiltonians with random two-body interactions (the socalled two-body random ensemble or TBRE) are very likely to yield a ground state with angular momentum $L=0$. In fact, the

\footnotetext{
*Present address: Facultad de Física, Universidad de Sevilla, Avda. Reina Mercedes s/n, E-4012 Sevilla, Spain.
}

probability for an $L=0$ state to become the ground state turns out to be much larger than that expected on the basis of the fraction of $L=0$ states in the model space. Similar regularities were later found to exist as well in bosonic [3] and electronic [4] many-body quantum systems with random interactions, so the occurrence of these regular phenomena in spite of the random nature of the two-body interactions seems to be a rather generic feature. The unexpected results of Johnson et al. are reminiscent of other statistical results associated with correlated sets of numbers, which are perceived as contrary to expectations. This is the case, for example, for Benford's law, which deals with the counterintuitive relative frequency distributions of digits in a given data set and which is related to scale invariance [5].

The observation of a statistical preference of $L=0$ ground states for ensembles of random two-body interactions has sparked a large number of investigations to further explore the properties of these random systems and to understand the mechanism for the emergence of regular ordered spectral features from random interactions [6-9]. The appearance of ordered spectra in systems with chaotic dynamics is a robust property that does not depend on the specific choice of the (two-body) ensemble of random interactions [2,10-12], timereversal symmetry [10], and the restriction of the Hamiltonian to one- and two-body interactions [13], nor is it limited to yrast states with small angular momentum $L=0,2,4$ [14] as used in the original studies [2,3]. Despite the progress and new insights in understanding the appearance of ordered spectra from random interactions, the proposed explanations offer partial solutions of the problem, without being able to account for all observed regular phenomena in systems of randomly interacting fermions and bosons. Among others, we mention induced pairing [15], geometric chaoticity for randomly interacting fermions $[7,16]$, mean-field analysis for the interacting boson model and the vibron model [17], spectral widths $[10,18]$, and an empirical method based on the eigenvalues of each independent two-body matrix element [12]. 
The robustness of the numerical results for both systems of randomly interacting fermions and bosons strongly suggests that an explanation of the origin of the observed regular features has to be sought in the many-body dynamics of the model space and/or the general statistical properties of random interactions, a conclusion that is suggested by many theoretical studies. In this respect, the approach of Chau et al. [19] for diagonal Hamiltonians stands out, since it makes use of the geometry of the model space, is valid for both fermions and bosons, and allows the calculation of the ground state probabilities exactly.

In this article, we show that the geometric analysis of Ref. [19] can be interpreted in terms of correlations (covariances) between energy eigenvalues to provide an entirely statistical explanation of the distribution of ground state angular momenta of randomly interacting many-body quantum systems. In addition, we show that the method can be extended to nondiagonal Hamiltonians by using perturbation theory.

\section{COVARIANCES}

In Ref. [19], spectroscopic properties of quantum systems with random interactions were given a geometric interpretation. In particular, it was shown that diagonal Hamiltonians, i.e., those whose energy eigenvalues depend linearly on the two-body matrix elements, can be associated with a geometric shape (convex polyhedron) defined in terms of coefficients of fractional parentage and/or generalized coupling coefficients. The probability for a certain state to become the ground state is then related to the angles at the vertices. In this approach, geometry arises as a consequence of strong correlations implicit in many-body quantum systems. Random tests can be understood in this context as sampling experiments on this geometry.

Let us consider many-body quantum systems for which the energy eigenvalues $e_{i}$ can be expressed as linear combinations of two-body matrix elements $r_{m}$ (as is the case for any interaction between identical fermions with $j \leqslant \frac{7}{2}$ or identical bosons with $l \leqslant 3$ ), that is,

$$
e_{i}=\sum_{m=1}^{d} c_{m}^{i} r_{m}
$$

The coefficients $c_{m}^{i}$ contain the information on the many-body quantum system via angular momentum coupling coefficients, coefficients of fractional parentage, etc. The statistical properties of such a system can be studied by taking an ensemble of random two-body matrix elements $r_{m}$ in which the random variables $r_{m}$ are chosen independently on a Gaussian distribution with zero mean and unit width. The covariance coefficients between two energies are given by

$$
\begin{aligned}
\left\langle e_{i} e_{j}\right\rangle & =\frac{1}{N-1} \sum_{p=1}^{N}\left(e_{i}^{(p)}-\left\langle e_{i}\right\rangle\right)\left(e_{j}^{(p)}-\left\langle e_{j}\right\rangle\right) \\
& =\sum_{s m=1}^{d} c_{m}^{i} c_{m}^{j}=\vec{c}^{i} \cdot \vec{c}^{j},
\end{aligned}
$$

where $N$ is the size of the ensemble and $p$ indicates the $p$ th realization of the ensemble. The covariances depend on the relative angle between the vectors $\vec{c}^{i}$ and $\vec{c}^{j}$.

Chau et al. showed that all energies are confined to a convex polytope (i.e., a convex polyhedron in $d$ dimensions) and that only the states located at the vertices of this polyhedron can become the ground state [19]. The probability for a state at vertex $j$ to become the ground state depends on the angle $\sum_{f \ni j} \theta_{j f}$, where the sum is over all faces that contain the vertex $j$, and $\theta_{j f}$ is the angle subtended at vertex $j$ in the face $f$. For $d=2$ and $d=3$ dimensions, the explicit forms are

$$
\begin{aligned}
& P_{j}^{(2)}=\frac{1}{2}-\frac{\theta_{j}}{2 \pi}, \\
& P_{j}^{(3)}=\frac{1}{2}-\frac{1}{4 \pi} \sum_{f \ni j} \theta_{j f} .
\end{aligned}
$$

From these considerations, it follows directly that the probability that a certain state can become the ground state is related to the covariances of the energies. Suppose that the angle $\theta_{j f}$ at vertex $j$ in the face $f$ is defined by the vertices $i, j$, and $k$ of the convex polygon. A straightforward application of the cosine rule shows that

$$
\cos \theta_{j f}=\frac{\left\langle e_{j}^{2}\right\rangle+\left\langle e_{i} e_{k}\right\rangle-\left\langle e_{i} e_{j}\right\rangle-\left\langle e_{j} e_{k}\right\rangle}{\sqrt{\left\langle e_{i}^{2}\right\rangle+\left\langle e_{j}^{2}\right\rangle-2\left\langle e_{i} e_{j}\right\rangle} \sqrt{\left\langle e_{k}^{2}\right\rangle+\left\langle e_{j}^{2}\right\rangle-2\left\langle e_{j} e_{k}\right\rangle}} .
$$

We thus find that the probability for a state that is located at a vertex $j$ of the convex polytope to become the ground state is determined by the covariance coefficients of the energy eigenvalues. It is important to note that this relation is exact and may be used to explain the approximate results based on the spectral widths $[10,18]$. Equation (4) is valid for any manybody quantum system whose energies are linear functions of the random variables and holds for both bosons and fermions.

\section{RESULTS}

The relation between the distribution of ground state angular momenta and the covariances between energy eigenvalues of Eq. (4) provides a completely statistical interpretation of the distribution of ground state angular momenta of randomly interacting many-body quantum systems. To the best of our knowledge, this is the first time such a connection has been derived in explicit form. Since the geometric method of Chau et al. is valid for diagonal interactions for which the energy eigenvalues depend linearly on the random interactions, Eq. (4) provides a statistical interpretation of the exact results of Ref. [19].

For systems in which one has both diagonal and offdiagonal matrix elements, the method of covariance coefficients cannot be applied directly, since the energy eigenvalues show a nonlinear dependence on the random variables. In this section, we show that also for nondiagonal systems the distribution of ground state angular momenta can be obtained by treating the off-diagonal interactions in perturbation theory. In this way, one obtains an approximate expression of the energy 
eigenvalues that is linear in the random variables and hence is amenable to a statistical analysis based on the covariances. Obviously, this is only valid as far as the distribution of ground state angular momenta is concerned. Realistic ground state wave functions have a far more complicated structure that goes way beyond the use perturbation theory.

As an illustration, we discuss two schematic Hamiltonians of the interacting boson model (IBM), a model for collective excitations in medium and heavy mass nuclei [20].

As a first example, we consider the IBM Hamiltonian

$$
H_{1}=\cos \chi d^{\dagger} \cdot \tilde{d}+\frac{\sin \chi}{4(N-1)}\left(s^{\dagger} s^{\dagger}-d^{\dagger} \cdot d^{\dagger}\right) \text { (h.c.), }
$$

which describes a transition between vibrational $(\sin \chi=0)$ and $\gamma$-unstable nuclei $(\cos \chi=0)$. In the general case, the Hamiltonian has to diagonalized numerically to obtain its eigenvalues. The eigenstates can be labeled by the total number of bosons $N$, the boson seniority $v$ and the angular momentum $l$. The ground state has either $v=0, v=1$, or $v=N$. In the present study, the angle $\chi$ is taken on the interval $-\pi<\chi \leqslant \pi$, so that all possible combinations of attractive and repulsive interactions are covered. For this reason, the Hamiltonian effectively depends on two random coefficients $\cos \chi$ and $\sin \chi$, which can be either attractive or repulsive.

The distribution of ground state angular momenta was obtained exactly in a mean-field study [17]. For even values of the number of bosons $N$, the ground state has $v=0$ in $75 \%$ of the cases and $v=N$ in the remaining $25 \%$. For odd values of $N$, the ground state is either $v=0, v=1$, or $v=N$ for $50 \%$, $25 \%$, and $25 \%$ of the cases, respectively.

The Hamiltonian of Eq. (5) has a spherical ground state for $\sin \chi \leqslant|\cos \chi|$ and a deformed one for $\sin \chi \geqslant|\cos \chi|[17,21]$. In the first case, the appropriate basis is that of the $U(5)$ limit of the IBM, $|N, n, v\rangle$. The first term in the Hamiltonian of Eq. (5) is diagonal in this basis, whereas the second term contains both a diagonal and a nondiagonal contribution. The latter interaction can be treated in first-order perturbation theory so that the approximate energy spectrum has a linear dependence on the parameters

$$
\begin{aligned}
E_{n v}= & \cos \chi n_{d}+\frac{\sin \chi}{4(N-1)}[(N-n)(N-n-1) \\
& +(n-v)(n+v+3)] .
\end{aligned}
$$

For the deformed solution, the appropriate basis is that of the $S O(6)$ limit of the IBM, $|N, \sigma, v\rangle$. In this case, the second term of Eq. (5) is diagonal, whereas the first term contains diagonal and off-diagonal contributions. In first-order perturbation theory, the energy spectrum is given by

$$
E_{\sigma v}=\sin \chi \frac{(N-\sigma)(N+\sigma+4)}{4(N-1)}+\cos \chi \sum_{n} n\left(\zeta_{n \sigma}^{v}\right)^{2},
$$

where $\zeta_{n \sigma}^{v}=\langle N, n, v \mid N, \sigma, v\rangle[22]$.

In Table I, we compare the exact results and the ones obtained in first-order perturbation theory, using the energy eigenvalues in Eqs. (6) and (7), and the correspondence between the covariances and distribution of ground state probabilities of Eq. (4). The results obtained in perturbation theory are remarkably close to the exact ones. There are some slight deviations for the spherical solutions, whereas in the
TABLE I. Distribution of ground state probabilities $P_{v}$ (in \%)

\begin{tabular}{|c|c|c|c|c|c|c|c|}
\hline \multirow[t]{2}{*}{$N$} & \multirow[t]{2}{*}{$v$} & \multicolumn{2}{|c|}{ Spherical } & \multicolumn{2}{|c|}{ Deformed } & \multicolumn{2}{|c|}{ Total } \\
\hline & & Full & Diag & Full & Diag & Full & Diag \\
\hline \multirow[t]{3}{*}{15} & 0 & 50.0 & 48.6 & 50.0 & 50.0 & 50.0 & 49.0 \\
\hline & 1 & 33.3 & 34.7 & 0.0 & 0.0 & 25.0 & 26.0 \\
\hline & $N$ & 16.7 & 16.7 & 50.0 & 50.0 & 25.0 & 25.0 \\
\hline \multirow[t]{3}{*}{16} & 0 & 83.3 & 83.3 & 50.0 & 50.0 & 75.0 & 75.0 \\
\hline & 1 & 0.0 & 0.0 & 0.0 & 0.0 & 0.0 & 0.0 \\
\hline & $N$ & 16.7 & 16.7 & 50.0 & 50.0 & 25.0 & 25.0 \\
\hline
\end{tabular}
for $N=15$ and $N=16$ bosons, obtained exactly (Full) and in the diagonal approximation (Diag) using Eq. (6) for the spherical solution and Eq. (7) for the deformed case.

deformed region there is an exact correspondence for the distribution of ground state angular momenta between the diagonal approximation and the full calculation.

The Hamiltonian of Eq. (5) is a special choice, since in addition to the number of bosons and the angular momentum, also the boson seniority $v$ is a conserved quantum number. Let us now consider a Hamiltonian in which this is not the case. As a second example, we take a schematic IBM Hamiltonian that describes the transition between vibrational and rotational nuclei. In the notation of Ref. [23], this Hamiltonian is given by

$$
\begin{aligned}
H_{2}= & \cos \chi d^{\dagger} \cdot \tilde{d}+\frac{\sin \chi}{N-1}\left[\left(2 s^{\dagger} s^{\dagger}-d^{\dagger} \cdot d^{\dagger}\right)(\text { h.c. })\right. \\
& \left.\left.+\left(2 s^{\dagger} d^{\dagger} \pm \sqrt{7} d^{\dagger} d^{\dagger}\right)^{(2)} \cdot \text { (h.c. }\right)\right] .
\end{aligned}
$$

The Hamiltonian of Eq. (8) has a spherical minimum for $-\pi<$ $\chi<\arctan \frac{1}{9}$ and a deformed minimum for $\arctan \frac{1}{9}<\chi \leqslant \pi$. For $\chi=\arctan \frac{1}{9}$, the system exhibits a first-order phase transition between spherical and deformed nuclei [21,23]. Just as in the previous example, the distribution of ground state angular momenta can be obtained exactly in a mean-field study. For $N=3 k(k=1,2, \ldots)$ the ground state has $L=0$ in $75 \%$ of the cases and $L=2 N$ (the maximum value of the angular momentum) in the remaining $25 \%$. For all other values of the total number of bosons $N$, the ground state has either $L=0, L=2$, or $L=2 N$ for $50 \%, 25 \%$, and $25 \%$ of the cases, respectively.

To test the correspondence between the covariances and the distribution of ground state angular momenta, we treat the Hamiltonian of Eq. (8) in first-order perturbation theory, in which the first term is treated as a perturbation to the second in the deformed region and vice versa in the spherical region. In Fig. 1, we compare the percentages of ground states with $L=0$ and $L=2$ obtained exactly and in first-order perturbation theory. Also in this case, the agreement is very good, although not at the level of precision as shown in Table I for the Hamiltonian of Eq. (5). The deviations observed for the probabilities for ground states with $L=0$ and $L=2$ may be reduced in second-order perturbation theory. The percentage of ground states with the maximum value of the angular momentum $L=2 \mathrm{~N}$ is $25 \%$ in all cases, both exactly and in perturbation theory. 


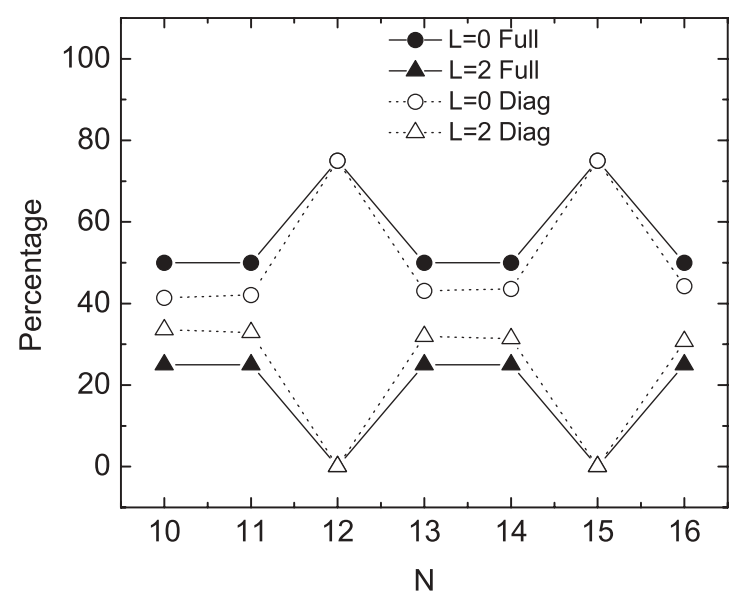

FIG. 1. Percentage of ground states with angular momentum $L=$ 0 (circles), and $L=2$ (triangles) for $10 \leqslant N \leqslant 16$ bosons interacting via the Hamiltonian of Eq. (8) with $-\pi<\chi \leqslant \pi$ calculated exactly (solid lines) and in first-order perturbation theory (dotted lines).

In both examples, the distribution of the ground state angular momenta is reproduced to a great level of precision with the present procedure, which is a combination of perturbation theory to get energy eigenvalues that are linear in the random two-body matrix elements, and the relation that we derived between the covariances and the probabilities of the ground state angular momenta.

The energies of many-body quantum systems are strongly correlated for the simple reason that they are all eigenvalues of the one and the same Hamiltonian. These strong correlations may modify the distribution of ground states from naive expectations, as was the case for the preponderance of $L=0$ ground states observed for calculations in the nuclear shell model and the IBM with random interactions [2,3]. Because of to the strong correlations, it should not be surprising that the probability distribution of the ground state angular momenta for an ensemble of random two-body interactions is related to the covariances between energy eigenvalues.

\section{SUMMARY AND CONCLUSIONS}

In this manuscript, we addressed the problem of the emergence of regular features in many-body quantum systems with random interactions. As an example, we focused on the probability distribution of ground state angular momenta in nuclear models, especially the unexpected observed dominance of $L=0$ ground states [2,3]. Even though many authors have tackled the subject, no general explanation for the preponderance of $L=0$ ground states has been found. A general feature of these systems is that the energy eigenvalues are strongly correlated, since all many-body matrix elements are expressed in terms of a relatively small number of random two-body matrix elements.

In this article, we proposed to explore the correlations between energy eigenvalues (or covariances) in more detail. We established, to the best of our knowledge for the first time, an explicit relation between the probability distribution of ground state angular momenta and the covariances between energy eigenvalues. For diagonal (but in no way trivial) Hamiltonians, our formulas in terms of covariance coefficients are exact. For nondiagonal Hamiltonians, it is not possible to give closed expressions, but by means of perturbation theory, nearly exact results can be found for the ground state percentages.

This new relation provides a purely statistical interpretation of the abundances of ground states with $L=0$ observed in numerical studies of many-body systems with random interactions which is, in contrast to all other explanations, valid for both fermions and bosons (e.g., the nuclear shell model and the IBM in nuclear physics). As an example of this procedure, we studied two schematic nondiagonal Hamiltonians of the IBM and found an excellent agreement between the approximate results obtained in perturbation theory and the exact ones. Although these results were illustrated in the context of a specific model, we believe them to be sufficiently general to propose an entirely statistical explanation that is valid in general for any many-body quantum system. In future work, we will apply the present method to problems in the nuclear shell model.

As a final comment, an explanation for the emergence of regular features in randomly interacting many-body systems which are based entirely on statistical arguments (covariances) may also provide a link to other unexpected results in statistical problems related to correlated data, such as Benford's and Zipf's laws.

\section{ACKNOWLEDGMENT}

This work was supported in part by Grant IN113808 of PAPIIT, UNAM, Mexico.
[1] F. Iachello and I. Talmi, Rev. Mod. Phys. 59, 339 (1987).

[2] C. W. Johnson, G. F. Bertsch, and D. J. Dean, Phys. Rev. Lett. 80, 2749 (1998).

[3] R. Bijker and A. Frank, Phys. Rev. Lett. 84, 420 (2000).

[4] Y. Alhassid, Rev. Mod. Phys. 72, 895 (2000).

[5] J. W. Wittaker, SIAM J. Appl. Math. 43, 257 (1983).

[6] R. Bijker and A. Frank, Nucl. Phys. News 11, No. 4, 15 (2001).

[7] V. Zelevinsky and A. Volya, Phys. Rep. 391, 311 (2004).

[8] Y. M. Zhao, A. Arima, and N. Yoshinaga, Phys. Rep. 400, 1 (2004).
[9] T. Papenbrock and H. A. Weidenmüller, Rev. Mod. Phys. 79, 997 (2007).

[10] R. Bijker, A. Frank, and S. Pittel, Phys. Rev. C 60, 021302(R) (1999).

[11] D. Dean, Nucl. Phys. A682, 194c (2001).

[12] Y. M. Zhao, A. Arima, and N. Yoshinaga, Phys. Rev. C 66, 034302 (2002).

[13] R. Bijker and A. Frank, Phys. Rev. C 62, 014303 (2000).

[14] C. W. Johnson and H. A. Nam, Phys. Rev. C 75, 047305 (2007).

[15] C. W. Johnson, G. F. Bertsch, D. J. Dean, and I. Talmi, Phys. Rev. C 61, 014311 (1999). 
[16] D. Mulhall, A. Volya, and V. Zelevinsky, Phys. Rev. Lett. 85, 4016 (2000).

[17] R. Bijker and A. Frank, Phys. Rev. C 64, 061303(R) (2001); 65, 044316 (2002).

[18] T. Papenbrock and H. A. Weidenmuller, Phys. Rev. Lett. 93, 132503 (2004); Phys. Rev. C 73, 014311 (2006).

[19] P. C. Huu-Tai, A. Frank, N. A. Smirnova, and P. V. Isacker, Phys. Rev. C 66, 061302(R) (2002).
[20] F. Iachello and A. Arima, The Interacting Boson Model (Cambridge University Press, Cambridge, England, 1987).

[21] A. E. L. Dieperink, O. Scholten, and F. Iachello, Phys. Rev. Lett. 44, 1747 (1980).

[22] O. Castaños, E. Chacón, A. Frank, and M. Moshinsky, J. Math. Phys. 20, 35 (1979); A. Arima and F. Iachello, Ann. Phys. (NY) 123, 468 (1979).

[23] A. E. L. Dieperink and O. Scholten, Nucl. Phys. A346, 125 (1980). 